\title{
Distribución actual y potencial de Dendroctonus mexicanus Hopkins bajo dos escenarios de cambio climático
}

\section{Actual and potential distribution of Dendroctonus mexicanus Hopkins under two climate change scenarios}

\author{
Fátima Monserrat Méndez Encina', Jorge Méndez González*1 y Julián Cerano Paredes²
}

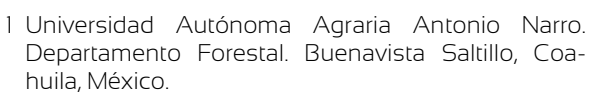

2 Instituto de Investigaciones Forestales, Agrícolas y * Autor de correspondencia. jmendezg@hotmail.com Pecuarias. CENID-RASPA. Gómez Palacio, Durango. México.

\section{RESUMEN}

El clima ejerce una función importante sobre la distribución natural de las especies. Los modelos bioclimáticos son una herramienta importante para evaluar la respuesta de las especies al cambio climático. El objetivo de este estudio fue estimar la distribución de Dendroctonus mexicanus Hopkins, bajo escenarios actuales y futuros (año 2050) de cambio climático y en dos valores (4.5 y 8.5) de trayectorias de concentración representativas (RCP’s). Los aspectos del modelo (creación, calibración, selección y evaluación) se realizaron con kuenm, un paquete de R con algoritmo de modelación Maxent. El modelo de idoneidad de D. mexicanus se constituyó principalmente de temperatura media del 1/4 anual más cálido (76.6\%), intervalo de temperatura anual (12.5\%) y precipitación de 1/4 anual más seco (5.5\%). En México, el área de idoneidad alta de D. mexicanus se calculó en $121310.7 \mathrm{~km}{ }^{2}$, de los cuales el Estado de México (17 879.7 km²), Michoacán (15 $\left.669.8 \mathrm{~km}^{2}\right)$, Puebla $\left(14693.1 \mathrm{~km}^{2}\right)$, Oaxaca $(14380.1 \mathrm{~km})^{2}$ e Hidalgo $(13830 \mathrm{~km}$ ), representaron más de $63 \%$ de esta superficie. Se estima que para el 2050, la idoneidad climática disminuya 51000 km², especialmente en Michoacán, Jalisco y Guanajuato y, en contraste, aumente cerca de $25000 \mathrm{~km}^{2}$ hacia el noroeste del país. Por lo que es factible una migración de este descortezador hacia mayores latitudes $(30 \mathrm{~km} \pm 15 \mathrm{~km})$ y altitudes $(\sim 100 \mathrm{~m})$, ampliando su registro hasta los $3700 \mathrm{~m}$ snm; estos cambios relevantes de su distribución pueden implicar daños forestales potenciales.

PALABRAS ClAVE: descortezadores, kuenm, Maxent, México, modelos bioclimáticos.

\section{ABSTRACT}

Climate exerts a dominant control over the natural distribution of species. Bioclimatic models are an important tool for assessing the potential responses of species to climate change. The objective of this study was to estimate the distribution of Dendroctonus mexicanus Hopkins, under current and future climate scenarios (year 2050) in two Representative Concentration Pathway (RCP's) 4.5 and 8.5. Characteristics of model (creation, calibration, selection and evaluation) were carried out in kuenm, an R package that uses Maxent as the modeling algorithm. Model suitability of D. mexicanus was composed mainly by mean temperature of warmest quarter (76.6\%), temperature annual range $(12.5 \%)$ and precipitation of driest quarter (5.5\%). In Mexico, the high suitability of D. mexicanus is 121310.7 $\mathrm{km}^{2}$; only five states: State of Mexico $\left(17879.7 \mathrm{~km}^{2}\right)$, Michoacan $\left(15669.8 \mathrm{~km}^{2}\right)$, Puebla $\left(14693.1 \mathrm{~km}^{2}\right)$, Oaxaca $(14380.1 \mathrm{~km} 2)$ and Hidalgo $\left(13830 \mathrm{~km}^{2}\right)$, comprise more than $63 \%$ of the estimated high suitability. For 2050 it is estimated a decrease of $51000 \mathrm{~km}^{2}$ of climatic suitability specially in Michoacan, Jalisco and Guanajuato, but an increase of about $25000 \mathrm{~km}^{2}$ toward northwest Mexico. A shift and a probable migration of this bark beetle toward higher latitudes $(30 \pm 15 \mathrm{~km})$ and altitudes $(\sim 100 \mathrm{~m})$ extending its distribution up to 3700 masl is feasible. These relevant changes in their probably distribution may involve potential forest damage.

KEYWORDs: bark beetles, kuenm, Maxent, México, Bioclimatic models. 


\section{INTRODUCCIÓN}

México tiene aproximadamente 138 millones de hectáreas de vegetación, lo que representa $70 \%$ de su territorio; de esa superficie, $17 \%$ corresponde a bosques templados (Comisión Nacional Forestal [Conafor], 2014). La función ecológica de este tipo de vegetación incluye la regulación del clima al actuar como sumideros de gases de efecto invernadero (Food and Agriculture Organization of the United Nations [FAO], 2018). La excesiva acumulación de estos gases en la atmósfera está provocando cambios en el clima, evidencia de ello es el aumento de temperatura de $0.87{ }^{\circ} \mathrm{C}$ registrado en el periodo de 2006 a 2015; de continuar esta tendencia, se podrían alcanzar los $1.5^{\circ} \mathrm{C}$ para al año 2040 (Allen et al., 2018). La alteración de la composición del ecosistema por la extracción de especies, la conversión de uso de suelo, y los incendios forestales, entre otros, provocan debilitamiento y estrés en los árboles, lo que reduce su eficiencia reguladora y favorece la incidencia de plagas forestales (Salinas et al., 2010).

Los insectos descortezadores son parte natural de la estructura y del funcionamiento de los bosques (Wood, 1982). No obstante, existe evidencia clara sobre la magnitud, frecuencia e intensidad de los brotes de descortezadores por efectos directos o indirectos del clima (Pureswaran, Roques y Battisti, 2018). En el caso de especies como Dendroctonus rufipennis Kirby y Dendroctonus ponderosae Hopkins, incrementarían su población bajo escenarios de calentamiento global (Bentz et al., 2010). Otros investigadores demuestran que con una temperatura de $1.6^{\circ} \mathrm{C}$, los descortezadores podrían: aumentar el número de generaciones por año (Logan y Powell, 2001; Waring et al., 2009), ampliar su distribución y modificar sus hábitos (Régnière, 2009; Kocmánková et al., 2010), además de incrementar su tasa de supervivencia al ubicarse en mayores latitudes y altitudes (Pureswaran et al., 2018).

Los modelos de distribución de especies relacionan las condiciones del ambiente con su posible distribución geográfica (Guisan y Zimmermann, 2000). A través del diagrama «BAM» pueden determinarse los factores causales de los cambios de distribución de una especie con base en: condiciones bióticas favorables ( $(\mathrm{B} »)$, condiciones abióticas («A») y las variables accesibles a la dispersión de la especie desde la región de su origen histórico («M») (Soberón y Nakamura, 2009; Soberón, Osorio-Olvera y Peterson, 2017). Para modelar la distribución de especies se han desarrollado distintos algoritmos tales como: BIOCLIM (Busby, 1991), GARP (Stockwell, 1999) y Maxent (Phillips, Dudík y Schapire, 2004). Este último es de los más robustos y el que mejor predice la idoneidad climática para las especies (Elith et al., 2006); también puede incluir un gran número de variables escenopoéticas (bioclimáticas) de diferente índole indicadas en el diagrama de «BAM» (Soberón et al., 2017) y que se han utilizado para modelar la distribución de las especies (Beaumont, Hughes y Poulsen, 2005).

El desarrollo de modelos en E.E.U.U. se ha enfocado en D. ponderosae (Régnière, 2009), Dendroctonus frontalis Zimmermann y $D$. ponderosae (Williams y Liebhold, 2002) y en Dendroctonus valens LeConte (Maldonado et al., 2015). Mientras que, en México, existe un trabajo relevante que modeló con BIOCLIM la distribución actual de 12 especies de descortezadores, entre ellas Dendroctonus mexicanus Hopkins, revelando que esta es la especie con más amplia distribución en los bosques de México y coloniza hasta 20 especies de pino (Salinas et al., 2010). Sosa et al. (2018), modelaron la distribución potencial de descortezadores a nivel género, mientras que Mendoza, Salinas, Olivo y Zúñiga (2011) hicieron lo propio con Dendroctonus rizophagus Thomas \& Bright, en la Sierra Madre Occidental. Por los impactos económicos, ecológicos y daños irreversibles que ocasiona a los ecosistemas, D. mexicanus es considerada la especie de descortezador más importante en México (Cibrián et al., 1995); de aquí la importancia de conocer su distribución actual y potencial.

A través del tiempo, se ha incrementado el número de registros de presencia de las diferentes especies, ampliando el conocimiento de sus límites de distribución (Armendáriz, Torres, López, Villa y Zúñiga, 2012), han mejorado los algoritmos de modelación (Cobos, Peterson, Barve y Osorio, 2019) y se han creado nuevas superficies bioclimáticas y modelos de circulación general (MCG's) 
(Karger et al., 2017), lo que da la pauta para generar más estudios sobre la distribución de especies.

\section{OBJETIVOS}

Los objetivos de este estudio fueron modelar la distribución actual y potencial de $D$. mexicanus, bajo diferentes escenarios de cambio climático para el año 2050, usando cinco MCG's: MIROC5, HadGEM2-AO, CSIRO-Mk3-6-0, CSM4 y CNRM-CM5 y dos «trayectorias de concentración representativas» (RCP) 4.5 y 8.5, así como conocer las variables climáticas asociadas a su presencia, los impactos del cambio climático sobre la distribución actual y futura de esta especie y posibles riegos de invasión. Se hipotetiza que esta especie es sensible al clima y por tanto su distribución geográfica será modificada al 2050.

\section{MATERIALES Y MÉTODOS}

\section{Área de estudio}

El estudio se llevó a cabo en el área de distribución actual de D. mexicanus, la cual se ubica en bosques de coníferas de México, que abarcan una superficie de 164422.7 km² (FAO, 2018), con predominancia de especies del género Pinus, Quercus, Juniperus y Abies (Flores y Gerez, 1994). Se localiza en las principales serranías montañosas, Sierra Madre Oriental (SMO), Occidental (SMOc), Eje Neolvolcánico Transversal (ENT), en altitudes de $50 \mathrm{~m}$ a $3500 \mathrm{~m}$ snm, temperaturas medias anuales entre $10{ }^{\circ} \mathrm{C}$ y $20{ }^{\circ} \mathrm{C}$ y precipitación de $600 \mathrm{~mm}$ a $1000 \mathrm{~mm}$ (Rzedowski, 1978).

\section{Obtención registros y variables bioclimáticas}

Los registros de presencia de $D$. mexicanus se obtuvieron del portal GBIF (Global Biodiversity Information Facility), de artículos científicos y de colectas propias. Los datos se depuraron con base en lo sugerido por Cobos, Jiménez, Nuñez, Romero y Simoes (2018), eliminando registros duplicados, de baja precisión, fuera del límite de la distribución de la especie (en altitud, latitud y longitud), con errores de identificación etc.; permitiendo al menos un registro por celda de las capas ambientales. La autocorrelación espacial entre los registros se eliminó en $\mathrm{R}$ usando una distancia de $5 \mathrm{~km}$ a través del paquete spThin (Aiello, Boria, Radosavljevic, Vilela y Anderson, 2015). Las variables bioclimáticas $\left(\sim 1 \mathrm{~km}^{2}\right.$ de resolución) utilizadas fueron las propuestas por Karger et al. (2017), excluyendo de las 19, Bio 8, Bio 9, Bio 18 y Bio 19 (Tabla 1) para disminuir la incertidumbre y por combinar información de precipitación y temperatura en la misma capa (Escobar, Lira, Medina y Peterson, 2014).

\section{Área de calibración y espacio ambiental}

La delimitación del área de calibración, denotada por «M» en el diagrama de «BAM», es fundamental en la modelación de nichos y distribución de especies, así como en la generación y transferencia del modelo (Soberón et al., 2017). Esta delimitación se realizó en ArcMap 10.1, aplicando un buffer de $70 \mathrm{~km}$ de radio al total de los registros $(\mathrm{n}=283$ ), identificando "outliers" tanto en el espacio geográfico («G») como en el espacio ambiental ( $(\mathrm{E} »)$, hasta definir «M»», aplicando la llamada «dualidad de Hutchinson» (Colwell y Rangel, 2009).

\section{Selección de variables bioclimáticas}

Las variables bioclimáticas se limitaron al área de calibración; para seleccionarlas, se realizó una modelación previa en Maxent 3.3.3k, con los parámetros predeterminados del programa (Phillips, Anderson y Schapire, 2006), usando todos los registros (50\% para entrenamiento y el resto para validación) para incluir toda la geografía y climas actuales ocupados por D. mexicanus. La importancia de cada variable se evaluó mediante pruebas jackknife, implementadas en Maxent (Phillips et al., 2006). En ArcMap 10.1, se realizó correlación de Pearson entre variables bioclimáticas en formato raster. Finalmente, la selección de las variables se realizó considerando la biología de la especie, el valor de importancia de la variable y el aporte individual y en conjunto hacia el modelo, además de elegir aquellas no correlacionadas entre sí $(r<0.8)$ (Elith $e t$ al., 2006). Tres grupos de variables fueron probados para modelar la distribución de D. mexicanus. Para evitar la sobrestimación del modelo y representaciones erróneas de los límites de la realidad biológica (Beaumont et al., 2005; Cobos et al., 2019), cada grupo se integró de cinco variables. 
TABLA 1. Variables bioclimáticas utilizadas para generación de modelos de distribución actual y potencial de Dendroctonus mexicanus Hopkins.

\begin{tabular}{|c|c|}
\hline Variable & Descripción \\
\hline Bio 2 & Intervalo medio diurno (media por mes [temp. máx. - temp. mín.]) \\
\hline Bio 3 & Isotermalidad (Bio 2 / Bio 7)*(100) \\
\hline Bio 4 & Estacionalidad de la temperatura (desviación estándar * 100) \\
\hline Bio 7 & Intervalo de temperatura anual (Bio 5 - Bio 6) \\
\hline Bio 10 & Temperatura media del $1 / 4$ anual más cálido $\left({ }^{\circ} \mathrm{C}\right)$ \\
\hline Bio 11 & Temperatura media del $1 / 4$ anual más frío $\left({ }^{\circ} \mathrm{C}\right)$ \\
\hline Bio 15 & Temporalidad de la precipitación (coeficiente de variación, \%) \\
\hline Bio 16 & Precipitación de 1/4 anual más húmedo (mm) \\
\hline Bio 17 & Precipitación de 1/4 anual más seco (mm) \\
\hline
\end{tabular}

\section{Calibración, creación y evaluación del modelo}

La calibración, creación y evaluación del modelo se realizó en Maxent, dentro de R Core Team (2019), utilizando el paquete kuenm (Cobos et al., 2019), el cual genera un gran número de modelos candidatos, evaluándolos de acuerdo con: significancia estadística, ROC parcial (curvas operadas por el receptor), tasas de omisión, criterio de información Aikaike o complejidad del modelo (AICc) (Phillips et al., 2006; Peterson, Papeş y Soberón, 2008) y pruebas de jackknife (Cobos et al., 2019). Se empleó ROC parcial para determinar el ajuste del modelo, excluyendo el estadístico AUC (Area Under Curve) ya que no se recomienda porque su predicción no ocupa todo el espectro del área de la curva ROC (Peterson et al., 2008).

\section{Transferencia de modelos y riesgo de extrapolación}

Los modelos calibrados se transfirieron en tiempo (al año 2050) y espacio (todo el mundo) a través de tres enfoques: i) sin extrapolación, ii) con extrapolación y iii) con clamping (Escobar et al., 2014), utilizando cinco MCG's: MIROC5, HadGEM2-AO, CSIRO-Mk3-6-0, CSM4 y CNRM-CM5, bajo dos RCP's: 4.5 y 8.5; cuyo análisis utilizó las medias de cada uno de ellos. El formato de salida fue de tipo logístico, donde los modelos resultantes (mapas) representan valores de idoneidad (0 - 1) de la especie (Phillips et al., 2006). El riesgo de extrapolación del modelo se realizó usando ExDet (Mesgaran, Cousens y Webber, 2014) basada en distancias de Mahalanobis, dentro del paquete ntbox de $\mathrm{R}$ (Osorio, Narayani, Soberón y Falconi, 2016).

\section{Idoneidad actual y futura}

Los mapas de idoneidad actual de D. mexicanus se estratificaron en tres categorías: alta, media y baja, usando el método de Dalenius y Hodges (1959), consistente en formar estratos lo más homogéneos posible (varianza mínima intra estrato y máxima inter estrato), buscando inicialmente qué distribución estadística se ajustó a los datos de idoneidad, esta fue obtenida en $\mathrm{R}$ con el paquete fitdistrplus (Delignette-Muller y Dutang, 2015) e integrada en Mathematica5, versión 5.0.0.0 y, posteriormente, se obtuvo la superficie por entidad federativa y por estrato. Los mapas de idoneidad actual y futura de D. mexicanus se 
transformaron a mapas binarios tomado como base el umbral de mínima presencia, después se analizaron mediante álgebra de rasters, para determinar: i) nuevas áreas idóneas en 2050, ii) sobreposición de áreas (actual y futura) y iii) áreas idóneas actuales que ya no lo son en 2050.

\section{Resultados}

Se obtuvieron 283 registros totales de ocurrencia de $D$. mexicanus, de los cuales, 67 se utilizaron para modelación. En «M», la Bio 1 se correlacionó positivamente $(0.20<\mathrm{r}<$ $0.95, \mathrm{p} \leq 0.05)$ con la mayoría de las variables, contrario a Bio 2, Bio 3 y Bio 15, que no se correlacionaron con las variables evaluadas. En «E», también llamado «espacio ambiental o modelo de nicho» (Fig. 1), se observan registros dispersos, indicando probablemente que D. mexicanus pudiera no encontrarse en equilibrio, o quizá a la falta de monitoreo de esta especie en ciertas regiones climáticas. En este modelo de nicho, la especie se registra desde -1.9 hasta $33.6^{\circ} \mathrm{C}$, con medias que van desde $9{ }^{\circ} \mathrm{C}$ hasta $21^{\circ} \mathrm{C}$ y desde $450 \mathrm{~mm}$ hasta $1755 \mathrm{~mm}$.

\section{Distribución actual de Dendroctonus mexicanus Hopkins}

Se generaron un total de 1479 modelos candidatos por kuenm (Cobos et al., 2019), reflejando todas las combinaciones de 17 multiplicadores de regularización, 29 tipos de respuesta de modelado y 3 distintos conjuntos de variables ambientales. Acorde con los estadísticos de selección, el modelo con mayor robustez fue el tipo de respuesta lineal+cuadrático (lq), con un regulizador 0.2 (Tabla 2) y el conjunto de variables Bio 3, Bio 7, Bio 10, Bio 11 y Bio 17. Los resultados muestran buen ajuste de los modelos de $D$. mexicanus, con valores de ROC parcial de 0.9314 (modelo de calibración) hasta 0.9485 (transferencia al 2050, RCP 4.5). La tasa de AUC fluctuó desde 1.8629 (calibración) hasta 1.8971 (transferencia al 2050, RCP 8.5).

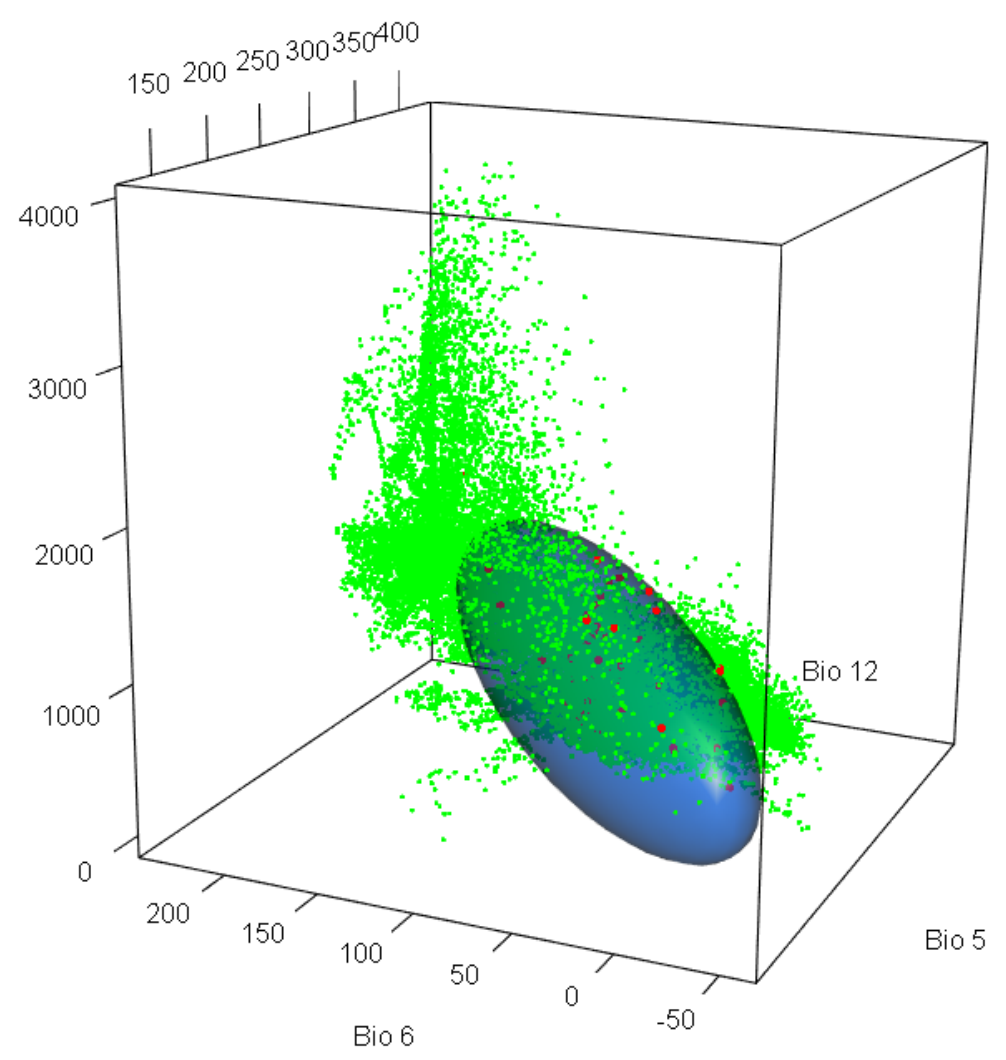

FIgURA 1. Modelo de nicho de Dendroctonus mexicanus Hopkins. 
TABLA 2. Estadísticas generales de los modelos candidatos de Dendroctonus mexicanus Hopkins.

\begin{tabular}{ll}
\hline Criterio & Número de modelos \\
\hline Total de modelos candidatos & 1479 \\
Modelos estadísticamente significantes (mES) & 1463 \\
Modelos que cumplen con el criterio tasa de omisión (mTO) & 15 \\
Modelos que cumplen con el criterio AICc (mAIC) & 1 \\
Número de mES y mTO & 15 \\
Número de mES y mAIC & 1 \\
Número de mES, mTO y mAIC & 1 \\
\hline
\end{tabular}

Independientemente del enfoque de la modelación (con extrapolación, sin extrapolación y clamping), los datos de idoneidad del modelo de distribución actual de D. mexicanus se ajustaron a una distribución Gamma. Los valores de los parámetros de 'forma' de la distribución Gamma fueron: 0.6003 y $2.7178 ; 0.6158$ y $2.1976 ; 0.5373$ y 1.6480 para el RCP 4.5 y 8.5 , y los valores de idoneidad fueron desde 0.0001 hasta $0.8309,0.8052$ y 0.8357 , respectivamente al enfoque. La idoneidad alta estimada para D. mexicanus, acorde con el enfoque sin extrapolación (Fig. 2a), representa $11.2 \%$ del total «M». Además, se distribuye en forma continua, principalmente en el Eje Neolvolcánico Transversal, destacando que en esta parte del país la idoneidad pasa directamente del estrato alto al bajo (Fig. 2a). La idoneidad media se observa hacia mayores latitudes, constituye $12.7 \%$ de «M», pero es discontinua. La modelación con los enfoques extrapolación (Fig. 2b) y clamping (Fig. 2c) muestran patrones geográficos similares al hacerlo sin extrapolación.

En México, el área de idoneidad alta de D. mexicanus es de $121310.7 \mathrm{~km}^{2}$; la idoneidad media es de $137710.4 \mathrm{~km}^{2}$ y la baja de $828710.2 \mathrm{~km}^{2}$. Las entidades: Estado de México (17 879.7), Michoacán (15 669.8), Puebla (14 693.1), Oaxaca (14 380.1) e Hidalgo $\left(13830 \mathrm{~km}^{2}\right)$, representan más de 63\% de la superficie de idoneidad alta estimada en «M» (Fig. 2a y 3). La idoneidad media ocurre en: Jalisco (18 672.47), Oaxaca (16 558.64) y Durango (15 $\left.173.74 \mathrm{~km}^{2}\right)$, equivalente a $37 \%$ del total de este estrato (Fig. 2a y 3).

\section{Perfil bioclimático actual y futuro de Dendroctonus mexicanus Hopkins}

Las variables bioclimáticas que mejor explican la distribución actual de D. mexicanus son las derivadas de temperatura: la media del 1/4 anual más cálido (Bio 10) y el intervalo de temperatura anual (Bio 7), contribuyendo con 76.6 y $12.5 \%$ respectivamente. La contribución de la Bio 10 es ligeramente mayor al modelar al $2050(\sim 80 \%$ en ambos RCP's), el resto de las variables (Bio 7, Bio 17, Bio 3 y Bio 11) aportan al modelo solo $23 \%$ (Tabla 3 ).

La variable de mayor contribución (Bio 10) muestra una distribución bimodal (Fig. 4a) en ambos periodos, actual y al 2050, y RCPS's. Sesenta por ciento de los registros de D. mexicanus se ubican actualmente en $18{ }^{\circ} \mathrm{C}$, acorde con la mediana de la distribución de frecuencias construida con $1+\left[1.33 \times \log _{10}(n)\right]$ propuesto por (Sturges, 1926). Se estima que, en las mismas zonas de registro, la temperatura aumente $1.6^{\circ} \mathrm{C}$ y $2.0^{\circ} \mathrm{C}$ en el año 2050 en RCP 4.5 y 8.5, respectivamente. En Bio 7, 78.8\% de los registros se ubican entre $17^{\circ} \mathrm{C}$ y $19{ }^{\circ} \mathrm{C}$ (Fig. 4b), el desplazamiento al 2050 es $0.8^{\circ} \mathrm{C}(\mathrm{RCP} 4.5)$ y $2.2^{\circ} \mathrm{C}(\mathrm{RCP}$ 8.5) más. Los MCG’s predicen al 2050 que la precipitación del 1/4 anual más seco (Bio 17) disminuirá entre 8.7\% y 19.1\% (RCP’s 4.5 y 8.5) en el área de distribución actual de D. mexicanus. En términos de precipitación $94.7 \%$ de los registros se ubican entre $10 \mathrm{~mm}$ y $40 \mathrm{~mm}$ (Fig. 4c) en Bio 17. En términos de altitud, $92.1 \%$ de los registros se ubican entre $1600 \mathrm{~m}$ y $2600 \mathrm{~m}$ snm (Fig. 4d). 


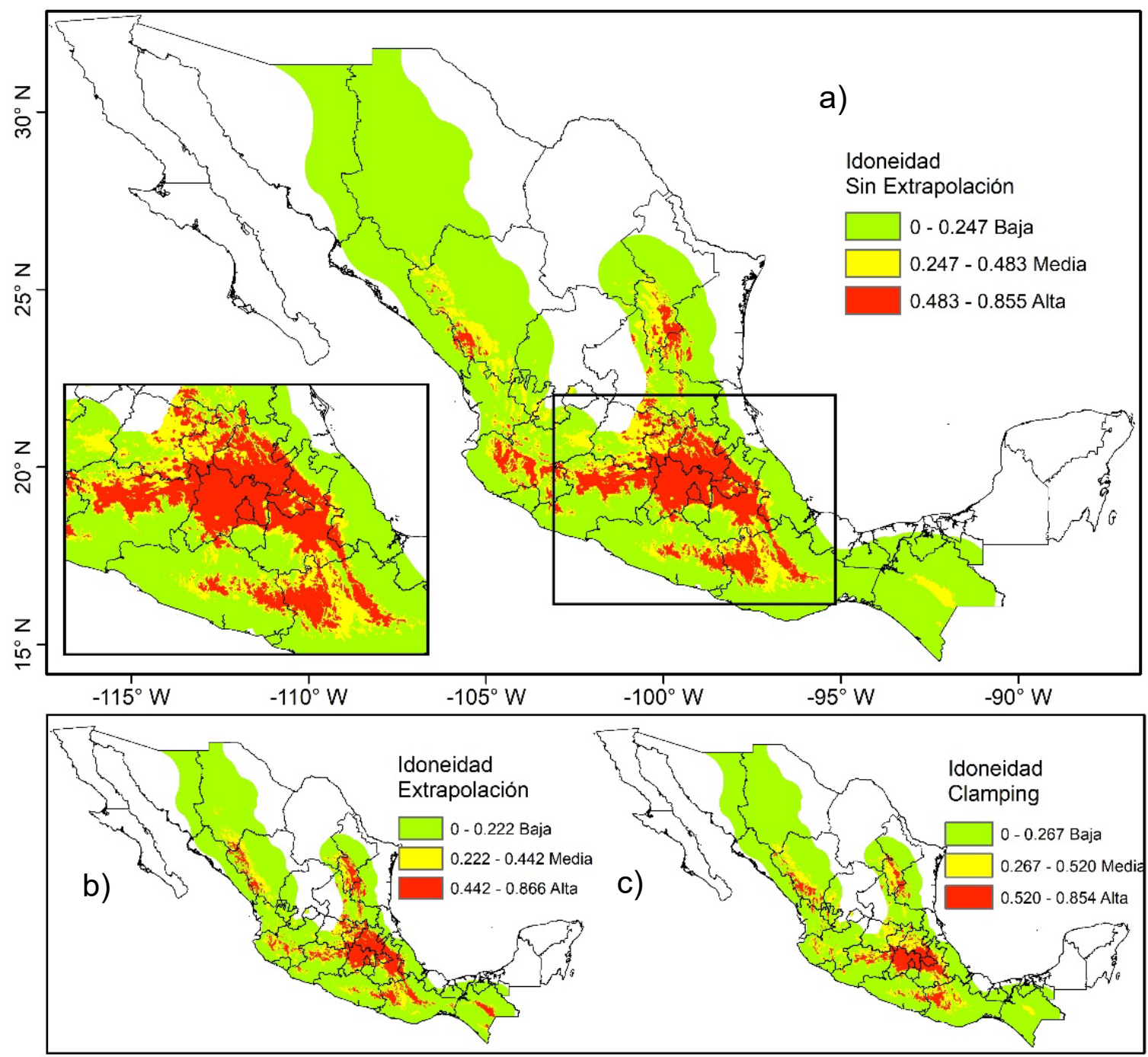

FIGURA 2. Áreas de idoneidad actual de Dendroctonus mexicanus Hopkins, estratificada en tres niveles (alto, medio y bajo) mediante la técnica de varianza mínima al interior de cada estrato y máxima entre ellos, en tres enfoques de modelación: sin extrapolación (a), con extrapolación (b) y con clamping (c).

TABLA 3. Contribución de las variables bioclimáticas al modelo actual, transferido al 2050 en dos trayectorias de concentración representativas RCPS’s 4.5 y 8.5 y perfil bioclimático de Dendroctonus mexicanus Hopkins.

\begin{tabular}{|c|c|c|c|c|c|c|}
\hline \multirow[b]{2}{*}{ Variable } & \multicolumn{3}{|c|}{ Contribución al modelo (\%) } & \multicolumn{3}{|c|}{ Perfil bioclimático (mínimo y máximo) } \\
\hline & Actual & RCP $4.5^{*}$ & RCP 8.5* & Actual" & RCP 4.5 & RCP 8.5 \\
\hline Bio 10 & 76.6 & $79.9 \pm 1.2$ & $80.58 \pm 0.6$ & $110.0-283.0$ & $126.6-300.0$ & $130.4-303.4$ \\
\hline Bio 7 & 12.5 & $11.1 \pm 0.8$ & $10.8 \pm 0.8$ & $134.0-275.0$ & $153.8-297.6$ & $141.4-279.4$ \\
\hline Bio 17 & 5.5 & $4.4 \pm 0.1$ & $4.32 \pm 0.3$ & $11.0-110.0$ & $9.0-100.6$ & $7.6-97.0$ \\
\hline Bio 3 & 2.3 & $3.1 \pm 0.9$ & $2.9 \pm 0.6$ & $442.0-590.0$ & $53.0-69.0$ & $48.2-63.8$ \\
\hline Bio 11 & 3.1 & $1.5 \pm 0.4$ & $1.4 \pm 0.5$ & 110.0 - 283.0 & $65.6-237.4$ & $69.8-240.8$ \\
\hline
\end{tabular}

¥Promedio \pm desviación estándar de cinco modelos de circulación general. "En las mismas unidades indicadas en tabla 1. 


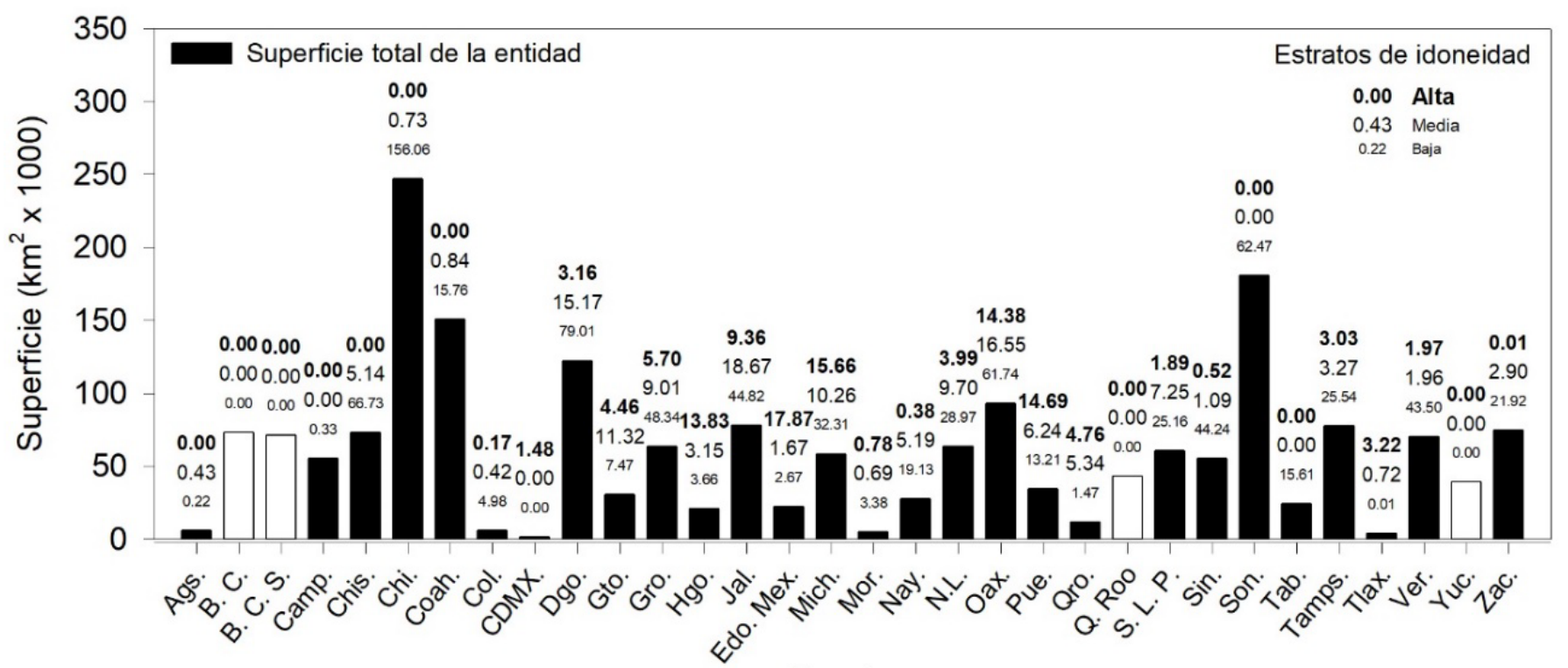

\section{Estados}

FIGURA 3. Superficie total por entidad federativa y estimada por estratos de idoneidad (baja, media y alta) de Dendroctonus mexicanus Hopkins.

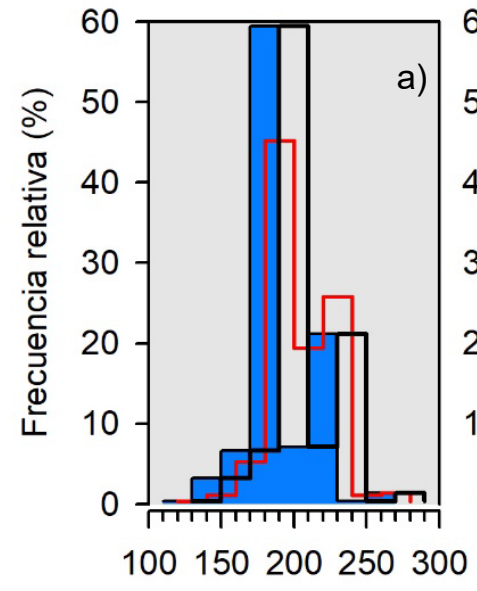

Bio $10\left({ }^{\circ} \mathrm{C} \times 10\right)$

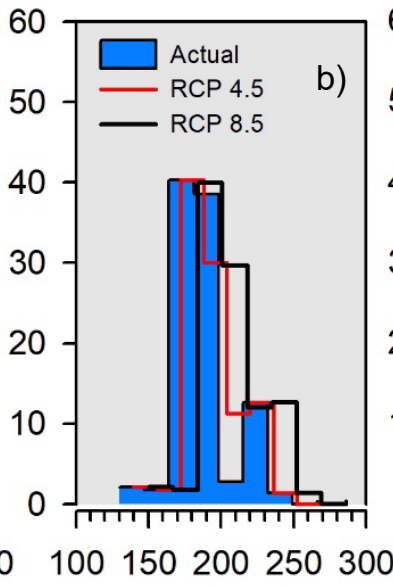

Bio $7\left({ }^{\circ} \mathrm{C} \times 10\right)$

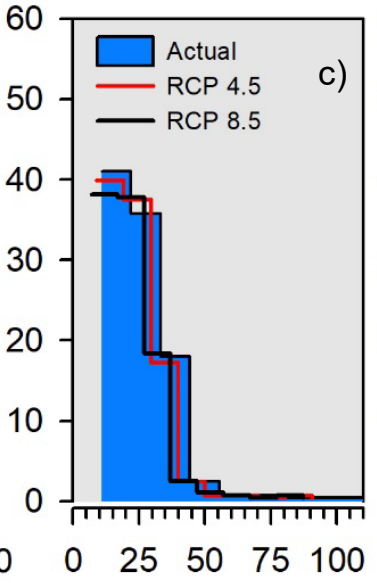

Bio $17(\mathrm{~mm})$

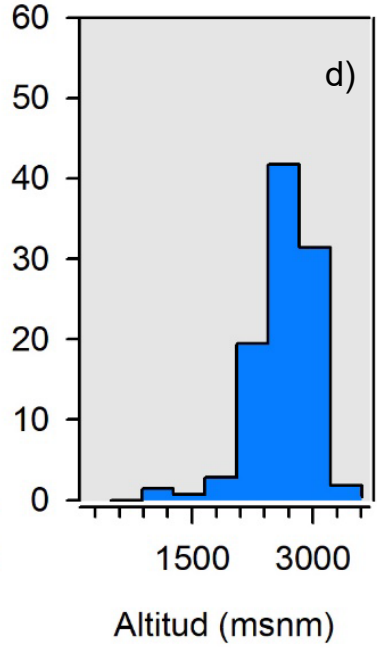

FIGURA 4. Histograma de frecuencia elaborado con 283 registros de Dendroctonus mexicanus Hopkins, distribuidos sobre tres variables ambientales de mayor contribución Bio 10 (a), Bio 7 (b) y Bio 17 (c), en el periodo actual (azul) y en el promedio de cinco Modelos de Circulación General (líneas) en dos trayectorias de concentración (RCP’s) 4.5 y 8.5 al 2050. Altitud (d). 


\section{Distribución potencial de Dendroctonus mexicanus Hopkins}

Al realizar la trasferencia del modelo actual a todas las regiones del mundo, se predicen nuevas áreas de idoneidad de D. mexicanus en México (climas análogos acorde a ExDet), e idoneidad, pero climas no análogos en Sudamérica (Bolivia) y en África (Etiopía, Angola y Sudáfrica, mapas no presentados). En ese sentido, la transferencia del modelo al 2050 en México muestra una posible expansión de D. mexicanus (color rojo, Fig. 5a), en total $24267 \mathrm{~km}^{2}$ y $25002 \mathrm{~km}^{2}$ estimadas para el RCP 4.5 y 8.5 respectivamente, superficies localizadas principalmente en la parte norte de la SMOc (Durango) y en menor proporción en la SMO (Nuevo León), lo que no se observó para el sur del país (Fig. 5a y 5b); esto implica una posible redistribución geográfica de $D$. mexicanus, migrando en promedio $30 \mathrm{~km} \pm 15 \mathrm{~km}$ hacia latitud norte y longitud este, únicamente en el norte del país.

Algunas áreas predichas actualmente como idóneas en el centro del país (color amarillo) podrían no serlo en el 2050; estas representan $47687 \mathrm{~km}^{2}$ y $51585 \mathrm{~km}^{2}$ para el RCP 4.5 y 8.5, respectivamente (Fig. 5 a y $5 b$ ). Al obtener la altitud en un total de 100000 puntos distribuidos aleatoriamente en los mapas de idoneidad actual y futura de $D$. mexicanus, reclasificados al umbral de 0.3639 (mínima presencia), se obtiene un promedio y desviación estándar de $2062 \mathrm{~m} \pm 444.51 \mathrm{~m}$ snm (distribución actual); al año
2050, los valores son de $2172 \mathrm{~m} \pm 434.5 \mathrm{~m}$ y $2180 \mathrm{~m} \pm$ $456.1 \mathrm{~m}$ snm para los RCP's 4.5 y 8.5 ; es decir, acorde con el promedio, esta especie podría migrar hasta $100 \mathrm{~m}$ en altitud para el 2050.

\section{DISCUSIÓN}

\section{Perfiles bioclimáticos}

El perfil bioclimático de las especies es de suma importancia para conocer sus tolerancias climáticas (Beaumont et al. 2005); aun así, pocos son los estudios que lo presentan. Mendoza et al. (2011) muestran que $D$. rhizophagus se distribuye entre $10.2^{\circ} \mathrm{C}$ y $19.2^{\circ} \mathrm{C}$ y desde 305 $\mathrm{mm}$ hasta $1406 \mathrm{~mm}$ de precipitación (obtenidos con Bio 1 y Bio 12). Con estas mismas variables bioclimáticas, se observa que el perfil de D. mexicanus es más amplio $\left(9^{\circ} \mathrm{C}\right.$ $21{ }^{\circ} \mathrm{C}$ ), incluso donde la precipitación alcanza $1700 \mathrm{~mm}$. Acorde con Bio 10 (variable que contribuye más al modelo de idoneidad de esta especie), $60 \%$ de los registros se presenta a una temperatura de $18{ }^{\circ} \mathrm{C}$, oscilando de $13{ }^{\circ} \mathrm{C}$ a $25^{\circ} \mathrm{C}$. Sin embargo, en D. valens (California, E.E.U.U.) la temperatura del mes más cálido (Bio 5) y la precipitación del mes más seco (Bio 14) explicaron su distribución potencial (Maldonado et al., 2015), mientras que la temperatura media (Bio 1) y altitud influyeron significativamente en la modelación de áreas idóneas de $D$. rhizophagus en el noroeste de México (Mendoza et al., 2011).
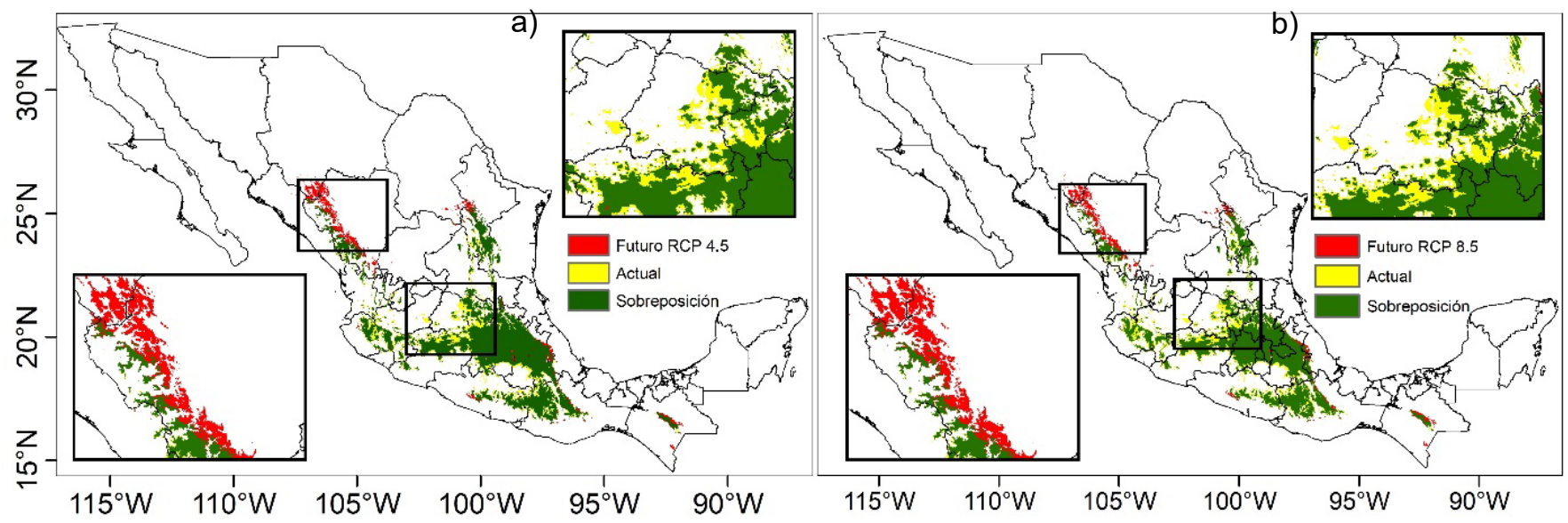

FIGURA 5. Áreas de idoneidad actual y futura de Dendroctonus mexicanus Hopkins para el año 2050, promedio estimado de cinco Modelos de Circulación General en dos trayectorias de concentración representativas (RCP), 4.5 (a) y 8.5 (b). 
En general, se observa que la temperatura es la variable que más influye en la distribución de especies de Dendroctonus spp. (Logan y Powell, 2001; Waring et al., 2009; Bentz et al., 2010; Pureswaran et al., 2018), como se ha evidenciado para esta especie; además, Varela, Lima-Ribeiro y Terribile (2015), indican que los modelos calibrados con variables de temperatura son más robustos comparados con aquellos que solo usan precipitación.

Los histogramas de frecuencias de las Bio 10 y Bio 7 (temperatura), construidos con $\mathrm{n}=283$, muestran posible ausencia de registros de D. mexicanus en los intervalos que van desde $19^{\circ} \mathrm{C}$ a $21{ }^{\circ} \mathrm{C}$ (Fig. 4a y 4b), mientras que el histograma de Bio 17 sigue una distribución sesgada a la derecha (Fig. 4c). Beaumont et al. (2005) demostraron que, si las variables poseen una distribución normal o sesgada, ejercen gran influencia en el perfil bioclimático de la especie, aunque construir un histograma debe tener una base estadística, puesto que puede manipularse dependiendo de cómo se determine el número de clases (Sturges, 1926).

\section{Modelos de idoneidad}

La distribución potencial de D. mexicanus en el presente estudio es semejante al modelo propuesto para Dendroctonus spp. en México (Sosa et al., 2018), el cual utilizó también Maxent, observándose gran similitud en las áreas de idoneidad predichas para D. mexicanus, demostrando que la mayor idoneidad de este género ocurre donde hay mayor diversidad de especies forestales y condiciones climáticas similares entre hospederos y hospedantes. De igual forma, se aprecian coincidencias con el modelo desarrollado por Salinas et al. (2010), el cual utilizó el algoritmo BIOCLIM, aunque dicha metodología no se recomienda por proporcionar predicciones imprecisas y ajustes bajos (Elith et al., 2006). Salinas et al. (2010) indican que 38\% de incidencias de D. mexicanus se presentan en Pinus leiophylla Schl. \& Cham., en este sentido, Aceves, Méndez, García y Nájera (2018) muestran que la Bio 10 es variable importante en determinar la distribución potencial de nueve especies de Pinus, incluyendo P. leiophylla, cuyo modelo de idoneidad es similar al de D. mexicanus, distribuyéndose en los mismos intervalos de temperatura desde $11.7^{\circ} \mathrm{C}$ hasta $21.1^{\circ} \mathrm{C}$ en Bio 10. Al modelar la distribución actual y futura de $P$. leiophylla, Cruz et al. (2016) revelan que esta es vulnerable al cambio climático, el modelo de idoneidad revela un decremento en su distribución en el sur de México, esto podría indicar que $D$. mexicanus busque nuevas especies hospederas.

\section{Migración altitudinal}

La distribución altitudinal actual de $D$. mexicanus de esta investigación es la misma que la señalada por Salinas et al. (2010) desde $800 \mathrm{~m}$ (Chiapas) hasta $3600 \mathrm{~m}$ snm (Estado de México), evidenciándose preferencia de esta especie hacia mayores altitudes (Fig. 4c). Bajo escenarios moderados (RCP 4.5) y drásticos (RCP 8.5) de emisiones de $\mathrm{CO}_{2}$ al 2050, se observa alta idoneidad climática para $D$. mexicanus, $100 \mathrm{~m}$ snm más allá del límite superior altitudinal actual, lo que supone que esta especie podría emigrar hacia esas altitudes. La preferencia de esta especie a migrar hacia mayores altitudes fue observada por Wood (1982) desde hace más de 30 años. Para Pinzón, Cuellar, Jurado y González (2018), la altitud resulta indicadora de la expansión de D. mexicanus, lo cual concuerda con los resultados obtenidos aquí. Régnière (2009) registró nuevos brotes de $D$. ponderosae en la parte occidental de Canadá, hacia mayores altitudes. Mendoza et al. (2011) muestran que D. rbizophagus tiene preferencias por hábitats más templados y por ende hacia mayores altitudes. En el noreste de E.E.U.U., por cada incremento de $1^{\circ} \mathrm{C}$, los brotes de $D$. ponderosae han incrementado $30.2 \mathrm{~m}$ en elevación (Williams y Liebhold, 2002).

\section{Migración latitudinal}

En este estudio se encontró que $D$. mexicanus podría desplazarse entre $15 \mathrm{~km}$ y $45 \mathrm{~km}$ hacia el Norte y Este, respecto a su distribución actual; es posible que el aumento de temperatura estimado entre $1.6^{\circ} \mathrm{C}$ y $2.0^{\circ} \mathrm{C}$ para el 2050 , en la temperatura media del 1/4 anual más cálido, sea el responsable de este movimiento. En otras especies de 
descortezadores, el desplazamiento geográfico de su distribución podría ser más amplio, e.g. Ungerer, Ayres y Lombardero (1999) muestran que, ante un aumento de 3 ${ }^{\circ} \mathrm{C}$ en la temperatura mínima anual, $D$. frontalis podría extender los límites de distribución de hasta $170 \mathrm{~km}$. Armendáriz et al. (2012) evidenciaron que D. rbizophagus ha ampliado su distribución geográfica $250 \mathrm{~km}$ hacia el Sur de la SMOc. En este mismo contexto, Régnière (2009) encontró evidencias de $D$. ponderosae sobre mayores latitudes, en la parte occidental de Canadá. Moser,
Fitzgibbon y Klepzig (2005) documentaron nuevos registros de D. mexicanus al sur de E.E.U.U. (Arizona), esto da evidencia de latitud más alta de esta especie; sin embargo, es importante resaltar que ningún grupo de variables empleadas aquí predijo idoneidad en la zona geográfica indicada por estos autores (Fig. 6a). La idoneidad de D. mexicanus en Sudamérica (Fig. 6b) no presenta clima análogo al área de calibración, según el error tipo 1 de ExDet (Fig. 6c), en especial Bio 3. Áreas idóneas, predichas por el modelo en el resto del mundo, tampoco presentan similitud climática.

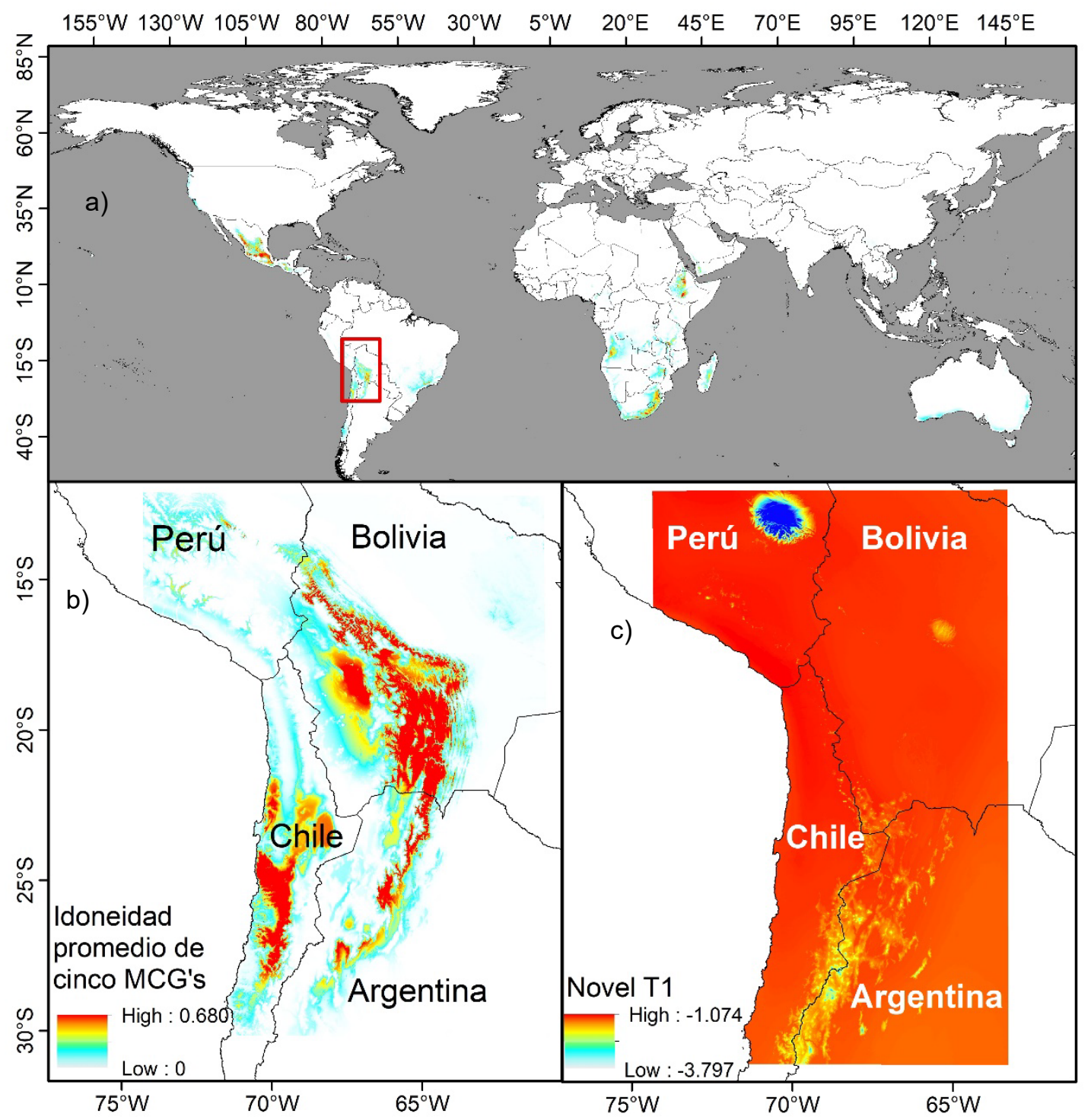

Figura 6. Áreas de idoneidad de Dendroctonus mexicanus Hopkins registradas en el mundo, obtenidas a través de la transferencia del modelo a), idoneidad de la especie predicha en Sudamérica b) y evaluación del riesgo de extrapolación de la idoneidad climática de la especie en Sudamérica, basada en distancias de Mahalanobis calculada con ExDet c). 
De la misma forma, Logan y Bentz (1999) sugieren que las especies de descortezadores podrían moverse hacia mayores elevaciones y más hacia el norte de su distribución. La revisión exhaustiva de Pureswaran et al. (2018) sobre insectos y cambio climático demuestra que los descortezadores tienen mayor tasa de supervivencia y reproducción al migrar hacia mayores altitudes y latitudes, se expanden hacia nuevas áreas, hospedando incluso nuevas especies, posiblemente porque los MCG's predicen los mayores incrementos de temperatura en mayores latitudes. Como se puede constatar en la literatura, la respuesta al calentamiento global será diferente entre y dentro de especies de descortezadores (Bentz et al., 2010).

\section{CONCLUSIONES}

Las variables que constituyeron el modelo de idoneidad de D. mexicanus fueron: temperatura de los meses más cálidos (Bio 10), oscilación entre la máxima y mínima (Bio 7), dentro de los meses con poca precipitación (Bio 17). Por la alta idoneidad predicha por el modelo, los estados en riesgo de ataque por esta especie son: Estado de México, Michoacán y Puebla. Bajo escenarios moderados (RCP 4.5) y drásticos (RCP 8.5) de emisiones de $\mathrm{CO}_{2}$, al 2050 se espera idoneidad de D. mexicanus en nuevas regiones en el norte del país $\left(24000 \mathrm{~km}^{2}\right.$ y $\left.25000 \mathrm{~km}^{2}\right)$, pero pérdida de áreas idóneas de casi el doble en el centro de México (47 $000 \mathrm{~km}^{2}$ y $51000 \mathrm{~km}^{2}$ ) respectivamente. Probable ampliación de su distribución altitudinal de hasta $100 \mathrm{~m}$ y un desplazamiento de entre $15 \mathrm{~km}$ y $45 \mathrm{~km}$ hacia la latitud Norte y longitud Este. Los resultados hacen suponer que un aumento de temperatura $\left(1.6^{\circ} \mathrm{C}-2.0^{\circ} \mathrm{C}\right.$ al 2050) en áreas de distribución actual de D. mexicanus podrían tener efectos importantes en la redistribución de esta especie, a pesar de poseer un perfil bioclimático más amplio que otras especies.

\section{RECONOCIMIENTOS}

A la Universidad Autónoma Agraria Antonio Narro por el apoyo financiero del proyecto de investigación número: 38111-0207-2334, del año 2019.

\section{REFERENCIAS}

Aceves R., L. D., Méndez G., J., García A., M. A., \& Nájera L., J. A. (2018). Distribución potencial de 20 especies de pinos en México. Agrociencia, 52(7), 1043-1057.

Aiello-Lammens, M. E., Boria, R. A., Radosavljevic, A. Vilela, B., \& Anderson, R. P. (2015). spThin: An R package for spatial thinning of species occurrence records for use in ecological niche models. Ecography, 38(5), 541-545. doi: 10.1111/ecog.01132

Allen, M. R., Dube, O. P., Solecki, W., Aragón-Durand F., Cramer, W., Humphreys, S., Kainuma, M., Kala, J., Mahowald, N., Mulugetta, Y., Perez, R., Wairiu, M., \& Zickfeld, K. (2018). Framing and Context. In: Global Warming of $1.5^{\circ} \mathrm{C}$. An IPCC Special Report on the impacts of global warming of $1.5^{\circ} \mathrm{C}$ above pre-industrial levels and related global greenhouse gas emission pathways, in the context of strengthening the global response to the threat of climate change, sustainable development, and efforts to eradicate poverty.

Armendáriz T., F., Torres B., V., López, M. F., Villa-Castillo, J., \& Zúñiga, G. (2012). New record and extension of the distribution range of the bark beetle Dendroctonus rbirophagus (Curculionidae: Scolytinae). Revista Mexicana de Biodiversidad, 83(3), 850-853. doi: $\underline{10.22201 / \mathrm{ib} .20078706 \mathrm{e} .2012 .3 .1261}$

Beaumont, L. J., Hughes, L., \& Poulsen, M. (2005). Predicting species distributions: Use of climatic parameters in BIOCLIM and its impact on predictions of species' current and future distributions. Ecological Modelling, 186(2), 251-270. doi: $\underline{10.1016 / \text { i.ecolmodel.2005.01.030 }}$

Bentz, B. J., Régnière, J., Fettig, C. J., Hansen, E. M., Hayes, J. L., Hicke, J. A., Kelsey, R. J., Negrón, J. F., \& Seybold, S. J. (2010). Climate change and bark beetles of the western United States and Canada: Direct and indirect effects. BioScience, 60(8), 602-613. doi: $\underline{10.1525 / \text { bio.2010.60.8.6 }}$

Busby, J. R. (1991). BIOCLIM - a bioclimate analysis and prediction system. Plant Protection Quarterly, 6(1), 8-9.

Cibrián T., D., Méndez M., J. T., Campos B., R., Yates, III. H. O., \& Flores-Lara, J. E. (1995). Insectos Forestales de México. Universidad Autónoma de Chapingo, Estado de México. Publicación no. 6, 43 p.

Cobos, M. E., Jiménez, L., Nuñez P., C., Romero A., D., \& Simoes, M. (2018). Sample data and training modules for cleaning biodiversity information. Biodiversity Informatics, 14, 49-50. doi: $\underline{10.17161 / \text { bi.v13i0.7600 }}$

Cobos, M. E., Peterson, A. T., Barve, N., \& Osorio O., L. (2019). kuenm: An $\mathrm{R}$ package for detailed development of ecological niche models using Maxent. PeerJ, 7, e6281. doi: 10.7717/peeri.6281 
Colwell, R. K., \& Rangel, T. F. (2009). Hutchinson's duality: the once and future niche. Proceedings of the National Academy of Sciences US A, 106, 19644-19650. doi: 10.1073/pnas.0901650106

Comisión Nacional Forestal [Conafor] (2014). Diagnóstico del Programa Presupuestario U036 PRONAFOR-Desarrollo Forestal 2014. Recuperado de https://www.coneval.org.mx/Informes/Evaluacion/Diagnostic o/Diagnostico 2014/Diagnostico 2014 SEMARNAT U036.p df

Cruz C., G., López M., L., Silva, J. T., Bernal S., N., Estrada G., F., \& López S., J. (2016). Potential distribution model of Pinaceae species under climate change scenarios in Michoacán. Revista Chapingo Serie Ciencias Forestales y del Ambiente, 22(2), 135-148. doi: 10.5154/r.rchscfa.2015.06.027.

Dalenius, T., \& Hodges, Jr. J. L. (1959). Minimum variance stratification. Journal of the American Statistical Association, 54(28), 88-101. doi: $\underline{10.1080 / 01621459.1959 .10501501}$

Delignette-Muller, M. L. \& Dutang, C. (2015). Fitdistrplus: An R package for fitting distributions. Journal of Statistical Software, 64(4), 1-34. doi: $10.18637 /$ jss.v064.i04

Elith, J., Graham, C. H., Anderson R. P., Dudik, M., Ferrier S., Guisan, A., Hijmans, R. J., Huettmann, F., Leathwick F., R. Leathwick J., Lehmann, A., Li, J., Lohmann, L. G., Loiselle, B. A., Manion, G., Moritz, C., Nakamura, M., Nakazawa, Y., Overton, J. McC., Peterson, A. T., Phillips, S. J., Richardson, K., Scachetti-Pereira R., Schapire, R. E., Soberon J., Williams, S., Wisz, Mary S., \& Zimmerman N. E. (2006). Novel methods improve prediction of species' distributions from occurrence data. Ecography, 29(2), 129_ 151. doi: $10.1111 /$ j.2006.0906-7590.04596.x

Escobar, L. E., Lira N., A., Medina V., G., \& Peterson, A. T. (2014). Potential for spread of the white-nose fungus (Pseudogymnoascus destructans) in the Americas: use of Maxent and NicheA to assure strict model transference. Geospatial Health, 9(1), 221-229. doi: $\underline{10.4081 / \text { gh. } 2014.19}$

Flores V., O. A., \& Gerez F., P. (1994). Biodiversidad y conservación en México: Vertebrados, vegetación y uso del suelo (2a ed.). Coyoacán, México: Conabio y UNAM.

Food and Agriculture Organization of the United Nations [FAO] (2018). El estado de los bosques del mundo. Las vías forestales hacia el desarrollo sostenible. Roma. doi: 10.18356/419ad9c6-es

Guisan, A., \& N. Zimmermann, E. (2000). Predictive habitat distribution models in ecology. Ecological Modelling, 135(2-3), 147-186. doi: $\underline{10.1016 / \mathrm{s} 0304-3800(00) 00354-9}$

Karger, D. N., Conrad, O., Böhner, J., Kawohl, T. Kreft, H., Soria-Auza, R. W., Zimmermann, N. E., Linder, H. P. \& Kessler, M. (2017).
Climatologies at high resolution for the earth's land surface areas. Scientific Data, 4, 170122. doi: 10.1038/sdata.2017.122

Kocmánková E., Trnka, M., Eitzinger, J., Formayer, H., Dubrovský, M., Semerádová, D., Žalud, Z., Juroch, J., \& Možný, M. (2010). Estimating the impact of climate change on the occurrence of selected pests in the Central European region. Climate Research, 44, 95-105. doi: $10.3354 / \operatorname{cr} 00905$

Logan, J. A., \& Bentz, B. J. (1999). Model analysis of mountain pine beetle (Coleoptera: Scolytidae) seasonality. Environmental Entomology, 28(6), 924-934. doi: 10.1093/ee/28.6.924

Logan, J. A., \& Powell, J. A. (2001). Ghost forests, global warming and the mountain pine beetle (Coleoptera: Scolytidae). American Entomologist, 47(3), 160-172. doi: 10.1093/ae/47.3.160

Maldonado M., J. D., Cera C., J. I., Mendoza A., R. A., Sáenz L., A. A., Torres O., M. E., Bravo P., L. C., \& Alotorre C. L., C. (2015). Distribución potencial de Dendroctonus valens mediante modelos de máxima entropía: Estado de California, E.U. Revista Lationomericana el Ambiente y las Ciencias, 6(12), 194-198.

Mendoza, Ma. G., Salinas M., Y., Olivo M., A., \& Zúñiga, M. G. (2011). Factors influencing the geographical distribution of Dendroctonus rbizophagus (Coleoptera: Curculionidae: Scolytinae) in the Sierra Madre Occidental, Mexico. Environmental Entomology, 40(3), 549559. doi: $10.1603 /$ en10059

Mesgaran, M. B., Cousens, R. D., \& Webber, B. L. (2014). Here be dragons: A tool for quantifying novelty due to covariate range and correlation change when projecting species distribution models. Diversity and Distributions, 20(10), 1147-1159. doi: $\underline{10.1111 / \text { ddi.12209 }}$

Moser, J. C., Fitzgibbon, B. A., \& Klepzig K. D. (2005). The Mexican pine beetle, Dendroctonus mexicanus (Coleoptera: Curculionidae): First record in the United States and co-ocurrence with southern pine beetle, Dendroctonus frontalis (Coleoptera: Curculionidae). Entomological News, 116(4), 235-243.

Osorio O., L., Narayani, B. Soberón, J., \& Falconi, M. (2016). Ntbox: From getting biodiversity data to evaluating species distributions models in a friendly GUI environment. R package version 0.2.5.4. https://rdrr.io/github/luismurao/ntbox/

Peterson, A. T., Papeş, M., \& Soberón, J. (2008). Rethinking receiver operating characteristic analysis applications in ecological niche modeling. Ecological Modelling, 213(1), 63-72. __ doi: 10.1016/i.ecolmodel.2007.11.008

Phillips, S. J., Anderson, R. P., \& Schapire, R. E. (2006). Maximum entropy modeling of species geographic distributions. Ecological Modelling, 190(3-4), 231-259. doi: $\underline{10.1016 / \text { i.ecolmodel.2005.03.026 }}$ 
Phillips, S. J., Dudík, M., \& Schapire, R. E. (2004). A maximum entropy approach to species distribution modeling. Twenty-First International Conference on Machine Learning - ICML '04, 83. doi: $10.1145 / 1015330.1015412$

Pinzón M., D., Cuellar R., L. G., Jurado, E., \& González T., M. (2018). Efecto de la sequía en la distribución y densidad de Dendroctonus mexicanus Hopkins, 1905 en bosques templados. Revista Mexicana de Ciencias Forestales, 9(50), 463-478. doi: 10.29298/rmcf.v9i50.256

Pureswaran, D. S., Roques, A., \& Battisti, A. (2018). Forest insects and climate change. Current Forestry Reports, 4(2), 35-50. doi: 10.1007/s40725-018-0075-6

R Core Team (2019). R: A language and environment for statistical computing. Vienna, Austria: R Foundation for Statistical Computing. Recuperado de https://www.R-project.org/

Régnière, J. (2009). Predicción de la distribución continental de insectos a partir de la fisiología de las especies, Unasylva, 60, 37-42. Recuperado de http://www.fao.org/3/i0670s/i0670s09.pdf

Rzedowski, J. (1978). Vegetación de México. México, D. F.: Limusa.

Salinas M., Y., Vargas M., C., Zúñiga, G., Victor J., Ager, A., \& Hayes, J. L. (2010). Atlas de distribución geográfica de los descortezadores del género Dendroctonus Curculionidae: Scolytinae) en México. México: Instituto Politécnico Nacional - Comisión Nacional Forestal.

Soberón, J., \& Nakamura, M. (2009). Niches and distributional areas: concepts, methods, and assumptions. Proceedings of the National Academy of Sciences, 106(Supplement 2), 19644-19650. doi: $\underline{10.1073 / \text { pnas. } 0901637106}$

Soberón, J., Osorio-Olvera, L., \& Peterson, T. (2017). Diferencias conceptuales entre modelación de nichos y modelación de áreas de distribución. Revista Mexicana de Biodiversidad, 88(2), 437-441. doi: 10.1016/i.rmb.2017.03.011

Sosa D., L., Méndez G., J., García A., M. A., Cambrón S., V., Villarreal Q., J. A., Ruiz G., C., \& Montoya J., J. C. (2018). Distribución potencial de barrenadores, defoliadores, descortezadores y muérdagos en bosques de coníferas de México. Revista Mexicana de Ciencias Forestales, 9(47), 187-208. _ doi: $\underline{10.29298 / \text { rmcf.v9i47.159 }}$

Stockwell, D. (1999). The GARP modelling system: Problems and solutions to automated spatial prediction. International Journal of Geographical Information Science, 13(2), 143-158. doi: $\underline{10.1080 / 136588199241391}$
Sturges, H. A., (1926). The choice of a class interval. Journal of the American Statistical Association, 21(153), 65-66.

Ungerer, M. J., Ayres, M. P., \& Lombardero, M. J. (1999). Climate and the northern distribution limits of Dendroctonus frontalis Zimmermann (Coleoptera: Scolytidae). Journal of Biogeography, 26(6), 1133-1145. doi: 10.1046/j.1365-2699.1999.00363.x

Varela, S., Lima-Ribeiro, M. S., \& Terribile, L. C. (2015). A short guide to the climatic variables of the last glacial maximum for biogeographers. PLOS ONE, 10(6). doi: 10.1371/journal.pone. 0129037

Waring, K. M., Reboletti, D. M., Mork, L. A., Huang, Chin-Hsun, Hofstetter R. W., Garcia, A. M., Fule', P. Z. \& Davis, T. S. (2009). Modeling the impacts of two bark beetle species under warming climate in the southwestern USA: Ecological and economic consequences. Environmental Management, 44(4), 824-835. doi: $\underline{10.1007 / \mathrm{s} 00267-009-9342-4}$

Williams, D. W., \& Liebhold, A. M. (2002). Climate change and the outbreak ranges of two North American bark beetles. Agricultural and Forest Entomology, 4(2), 87-99. doi: 10.1046/j.1461$9563.2002 .00124 . \mathrm{x}$

Wood, S. L. (1982). The bark and Ambrosia beetles of North and Central America (Coleoptera: Scolytidae), A taxonomic monograph. Utah, United States: Brigham Young University.

\section{Manuscrito recibido el 15 de julio de 2019}

Aceptado el 3 de enero de 2020

Publicado el 10 de junio de 2020

Este documento se debe citar como:

Méndez E., F. M., Méndez G., J., y Cerano P., J. (2020). Distribución actual y potencial de Dendroctonus mexicanus Hopkins bajo dos escenarios de cambio climático. Madera y Bosques, 26(2), e2622020. doi: 10.21829/myb.2020.2622002

Madera y Bosques por Instituto de Ecología, A.C. se distribuye bajo una Licencia Creative Commons Atribución-NoComercialCompartirlgual 4.0 Internacional. 